\title{
Raman scattering in crystals with order-disorder type phase transition
}

\author{
Ya.Ivankiv \\ Institute for Condensed Matter Physics \\ of the National Academy of Sciences of Ukraine, \\ 1 Svientsitskii Str., 79011 Lviv, Ukraine
}

Received October 14, 1998

The theory based on the "operator" two-time Green functions formalism applied for the construction of the polarizability operators entering the general expression for the effective cross-section of Raman scattering is developed. The presented microscopic scheme is applied to the investigation of the ferroelectrics with hydrogen bonds.

Key words: Raman scattering, order-disorder phase transition

PACS: 77.84.Fa, 78.30.Ly

\section{Raman scattering tensor}

The main characteristics of Raman scattering is the effective cross-section which can be expressed by Raman tensor [1]

$$
\frac{1}{2 \pi} \int_{-\infty}^{+\infty} \mathrm{d} t \mathrm{e}^{i\left(\omega_{1}-\omega_{2}\right) t}\left\langle\hat{P}_{\overrightarrow{k_{2},-k_{1}}}^{\beta^{\prime} \alpha^{\prime}}\left(-\omega_{1}, t\right) \hat{P}_{-\overrightarrow{k_{2}, k_{1}}}^{\beta \alpha}\left(\omega_{1}, 0\right)\right\rangle=H_{\beta^{\prime} \alpha^{\prime}, \beta \alpha}\left(\omega_{1} \omega_{2}\right)
$$

where

$$
\hat{P}_{\overrightarrow{k_{2}},-\overrightarrow{k_{1}}}^{\beta^{\prime} \alpha^{\prime}}\left(-\omega_{1}, t\right)=\mathrm{i} \int_{-\infty}^{t} \mathrm{~d} s\left[\hat{M}^{\beta^{\prime}}\left(\overrightarrow{k_{2}}, t\right), \hat{M}^{\alpha^{\prime}}\left(-\overrightarrow{k_{1}}, s\right)\right] \mathrm{e}^{\mathrm{i} \omega_{1}(s-t)}
$$

is the polarizability operator; $\omega_{1}, \omega_{2}$ are frequencies; $\overrightarrow{k_{1}}, \overrightarrow{k_{2}}$ are wave vectors of the incident and the scattered light, respectively; $\alpha, \beta, \alpha^{\prime}, \beta^{\prime}$ are coordinate indices.

Here, $\hat{M}^{\beta}(\vec{k})=\sum_{n} \mathrm{e}^{\mathrm{i} \vec{k} \vec{R}_{n}} \hat{M}_{n}^{\beta}$ are the Fourier transforms of the dipole momenta operators of the unit crystal cell ( $n$ is the cell number).

$$
\hat{\vec{M}}_{n}=\sum_{k}\left(e \hat{\vec{D}}_{n k}+z_{k} \vec{U}_{n k}\right) \equiv \sum_{k} \hat{\vec{M}}_{n k}
$$


where $\hat{\vec{D}}_{n k}$ are the electronic operators; $\vec{U}_{n k}$ are the ionic displacements. The summation in (1.3) is fulfilled over ionic species in the cell $\left(z_{k}\right.$ is the ionic charge, $k$ is the ionic type index); in (1.2) the operators are given in Heisenberg representation. In the semiphenomenological description of the Raman scattering the polarizability operators are presented as the expansions in vectors of ionic displacements. Equation (1.2) enables us to obtain the explicit form for the operator $\hat{P}_{-\vec{k}_{2}, \vec{k}_{1}}^{\beta \alpha}$; its specific features are determined by the Hamiltonian of the system. In the following we use the polarizability operator presented in terms of the "operator" Green functions [2]:

$$
\hat{G}_{\hat{A} \hat{B}}(t, s) \equiv\{\{\hat{A}(t) \mid \hat{B}(s)\}\}=-\mathrm{i} \theta(t-s)[\hat{A}(t), \hat{B}(s)] .
$$

where $\hat{A}(t), \hat{B}(s)$ are the operators written in Heisenberg representation. The statistical averaging of the $\hat{G}_{\hat{A} \hat{B}}(t, s)$ operator leads to the retarded two-time Green function $\langle\langle\hat{A}(t) \mid \hat{B}(s)\rangle\rangle$.

After the Fourier transformation we have

$$
\hbar \omega_{1}\{\{\hat{A} \mid \hat{B}\}\}_{\omega_{1}, \omega_{2}}=\frac{\hbar}{2 \pi}[\hat{A}, \hat{B}]_{\omega_{1}-\omega_{2}}+\{\{[\hat{A}, \hat{H}] \mid \hat{B}\}\}_{\omega_{1}, \omega_{2}} .
$$

In the presence of small parameters, the equation of the (1.5) type makes it possible to construct the operator expansions for the Fourier transformants $\{\{\hat{A} \mid \hat{B}\}\}_{\omega_{1}, \omega_{2}}$.

By means of (1.3) and (1.4) the expression (1.2) for the polarizability operator may be presented as follows

$$
\hat{P}_{\vec{k}_{2},-\vec{k}_{1}}^{\beta^{\prime} \alpha^{\prime}}\left(-\omega_{1}, t\right)=-2 \pi \sum_{n n^{\prime}} \sum_{l l^{\prime}} \mathrm{e}^{\mathrm{i} \vec{k}_{2} \vec{R}_{n}} e^{-\mathrm{i} \vec{k}_{1} \vec{R}_{n^{\prime}}} \cdot \int_{-\infty}^{+\infty} \mathrm{d} \omega_{1}^{\prime}\left\{\left\{\hat{M}_{n l}^{\beta^{\prime}} \mid \hat{M}_{n^{\prime} l^{\prime}}^{\alpha^{\prime}}\right\}\right\}_{\omega_{1}^{\prime},-\omega_{1}} \mathrm{e}^{-\mathrm{i}\left(\omega_{1}+\omega_{1}^{\prime}\right) t} .
$$

Thus, the problem of the explicit expression for the polarizability operator is reduced to determining the Fourier transformant $\{\{\hat{M} \mid \hat{M}\}\}_{\omega_{1}^{\prime},-\omega_{1}}$.

\section{Raman scattering on internal vibrations of ionic groups in crystals of $\mathrm{KH}_{2} \mathrm{PO}_{4}$}

In the problem of Raman scattering by the crystals with a complicated structure, we dwell upon the scattering by the internal vibrations of ionic groups which can be in different configurations. Configurations are considered to mean different location of the structural elements which form an ionic group including the structural elements which can be ordered at phase transition. In the case when the vibrations of internal and external (translational and librational) modes differ essentially they may be considered independently.

Consider the case when characteristics of vibrations and related electron states depend on the configurations of some of the ionic complexes in the unit cell. Then 
the Hamiltonian is written:

$$
\hat{H}=\sum_{n k} \sum_{i}\left(\sum_{s_{i}} \lambda_{k s_{i}}^{(i)} \hat{X}_{n k}^{s_{i} s_{i}}\right) N_{n k}^{i i}-\sum_{\substack{n k \\ \nu \alpha}} \sum_{i}\left(\sum_{\substack{s_{i} s_{i}^{\prime} \\ k \nu \alpha}} \theta_{i s_{i}^{\prime}}^{s_{i}} \hat{X}_{n k}^{s_{i} s^{\prime}} U_{n k \nu}^{\prime i \alpha}\right) N_{n k}^{i i}+\hat{H}_{\mathrm{ph}}
$$

Here, $N_{n k}^{i i}$ is the occupation number of the i-th configuration of the complex $(\mathrm{n}, \mathrm{k})$; $\hat{X}_{n k}^{s_{i} s_{i}^{\prime}}$ are the Hubbard operators (see [3]) acting based on the electron states of the groups; $\lambda_{k s_{i}}^{(i)}$ denotes energy of the state $s_{i}$ of the group k. We confine ourselves to the account of electron-vibrational interaction within individual ionic groups; $U_{n k v}^{\prime}$ are the vectors of ion displacement from equilibrium positions ( $\nu$ is the number of the ion in a group); $\theta_{k \nu \alpha}^{s_{i} s_{i}^{\prime}}$ is the interaction matrix. Beside the terms in (2.1) the total Hamiltonian includes the interaction energy of ionic groups (dependent on their configurations) which is responsible for the transition to the order state (in the case of the crystal possessing the order-disorder type phase transition).

Expression for the scattering cross-section can be written in terms of the Green function constructed of the polarizability operators [4].

In order to describe the one phonon Raman scattering by internal vibrations of ionic groups in the ferroelectric crystals with hydrogen bonds we apply configurational averaging and the approach of mean field type. As an example we consider the crystals of $\mathrm{KH}_{2} \mathrm{PO}_{4}$ (KDP)-type. In such crystals the various configurations of $\mathrm{H}_{n} \mathrm{PO}_{4}$ groups $(\mathrm{n}=0,1, \ldots, 4)$ can be realized depending on the character of proton location in the double potential wells on the hydrogen bonds. The most probable between them are the configurations with two protons in the neighbourhood of the given complex $\mathrm{PO}_{4}[5,6]$. Confine ourselves to the account for such configurations only; their number is six, more preferable energetically are two of them with protons near the upper or lower (with respect to the crystal c-axis) oxygen atoms (figure 1). We consider the case $T>T_{\mathrm{c}}$.

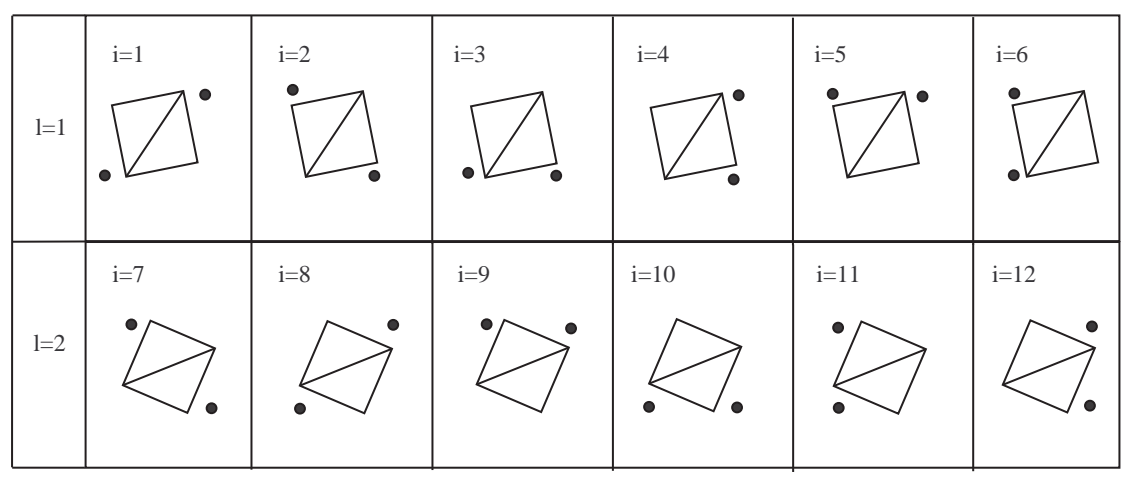

Figure 1. $\mathrm{H}_{2} \mathrm{PO}_{4}$ configurations for two sublattices of $\mathrm{PO}_{4}$ groups in KDP crystal (projection to xy-plane). 
For isolated $\mathrm{PO}_{4}$ complex of the point symmetry $T_{d}$ the internal vibrations are classified in terms of irreducible representations $A_{1}$ ( $\nu_{1}$ frequency); $E$ ( $\nu_{2}$ frequency); $F_{2}\left(\nu_{3}\right.$ and $\nu_{4}$ frequencies). In the case of configurations $\mathrm{i}=1,2 \quad(\mathrm{l}=1), \mathrm{i}=7,8 \quad(\mathrm{l}=2)$ they reduce to the modes of the symmetry $A_{1}, B_{1}, B_{2} ; 2 A_{1}, 2 B_{1}, 2 B_{2} ; A_{1}, B_{1}$, $B_{2}, 4 E$ respectively, and for configurations $\mathrm{i}=3, \ldots, 6(\mathrm{l}=1)$ and $\mathrm{i}=9, \ldots, 12(\mathrm{l}=2)$ into the modes with symmetry $A_{1}, B_{1}, B_{2}, 2 E ; 2 A_{1}, 2 B_{1}, 2 B_{2}, 4 E ; 3 A_{1}, 3 B_{1}, 3 B_{2}, 6 E$. Splitting of the frequencies $\nu_{2}, \nu_{3}, \nu_{4}$ is insignificant when symmetry lowers and its value is not established experimentally, although it is quite possible that the splitting of the mode $\nu_{3}$ observed in [7] is configurational. The electronic spectrum of $\mathrm{PO}_{4}$ groups is more sensitive to the location of the nearest proton.

The configurationally averaged expression for the effective Raman scattering cross-section contains contributions from different configurations of $\mathrm{H}_{n} \mathrm{PO}_{4}$ groups. If the correlation corrections are neglected the specific weights of such contributions are proportional to the mean occupation numbers $\left\langle N_{l}^{i i}\right\rangle$ and to the scattering tensor elements and are determined by the proton distribution on the hydrogen bonds [8].

The possible situations for $\mathrm{H}_{2} \mathrm{PO}_{4}$ groups with account of the contributions of different configurations are presented on table 1 ; the symmetry of phonon modes arising due to both configurational and Davydov splitting is shown.

Since $\mathrm{H}_{2} \mathrm{PO}_{4}$ configurations possess lower symmetry as compared to the $S_{4}$ symmetry of the position of $\mathrm{PO}_{4}$ groups in the crystal, it is possible that the lines may appear which are forbidden by the site symmetry. In particular, such a situation takes place at the $(\mathrm{zz})$ - scattering $\left(\mathbf{e}_{1}\left|\mathbf{o z}, \mathbf{e}_{2}\right| \mathbf{o z}\right)[9]$. As it follows from table 1 , at a given scattering geometry, besides the lines with $\nu_{1}$ and $\nu_{2}$ frequencies, the lines $\nu_{3}$ and $\nu_{4}$ should appear too (that are missing both for $T_{d}$ symmetry and for $S_{4}$ ). This result agrees with experimental data [10]; $\nu_{3}$ and $\nu_{4}$ lines that are observed in Raman scattering spectra are considerably weaker in comparison with the principal ones and appear at the temperatures near $T_{c}$ only.

The lines forbidden by the symmetry of $\mathrm{PO}_{4}$ groups may appear also in the cases of (xy) - scattering $\left(\nu_{1}\right.$ and $\nu_{2}$ frequencies $)$, of $(\mathrm{xz})-$ or $(\mathrm{yz})$ - scattering ( $\nu_{1}$ and $\nu_{2}$ frequencies $)$ and of $(\mathrm{xx})-$ or $(\mathrm{yy})-$ scattering $\left(\nu_{3}, \nu_{4}\right.$ frequencies $)$. The former of these cases for $\nu_{1}$ frequency is investigated experimentally in [10]. The second one is of interest, because "lateral" $\mathrm{H}_{2} \mathrm{PO}_{4}$ configurations contribution are only $(\mathrm{i}=3, \ldots 6,9, \ldots, 12)$. This is investigated experimentally in [11]. One of the peaks of Raman intensity, observed in this work, may correspond to the frequency $\nu_{1}$. However authors of this work connect this peak to the splitted mode $\nu_{3}$ and think, that these "lateral" configurations of the symmetry $C_{1}$ do not manifest itself in the Raman scattering spectra.

As to the possible usage of the proton ordering model for describing the ferroelectric phase transition in crystals of KDP group the attention should be paid to a more thorough experimental investigation of the cases (see table 1) when "lateral" configurations of protons, possessing $C_{1}$ symmetry, appear in the scattering since the model given allows for the existence of this kind of configuration (and configurations with different proton number). This problem is of importance in connection with the possibility of another interpretation of the phase transition in $\mathrm{KH}_{2} \mathrm{PO}_{4}$ which 
Table 1. Scattering geometry of the configurationally splitted modes $\left(T>T_{\mathrm{c}}\right.$ case). First column is the Davydov splitting. Fourth column is the configurational splitting. Symbols * denote the presence of non-zero components of scattering tensors; $\otimes$ correspond to the scattering in the case of isolated $\mathrm{PO}_{4}$ complexes of $T_{\mathrm{d}}$ symmetry. Two last columns show the symmetry of modes arising from different configurations.

\begin{tabular}{|c|c|c|c|c|c|c|c|c|c|c|c|}
\hline $\begin{array}{l}D_{2 \alpha} \\
\left\{\begin{array}{c}k \\
\bar{x}\end{array}\right\}\end{array}$ & $\begin{array}{c}S_{4} \\
\{\bar{x}\}\end{array}$ & $\begin{array}{l}T_{\alpha} \\
\{\bar{x}\}\end{array}$ & $\begin{array}{c}D_{2 \alpha} \\
\left\{\begin{array}{c}k(i) \\
\bar{x}_{i}\end{array}\right\}\end{array}$ & $(\mathrm{xx})$ & (yy) & (zz) & $(x y)$ & $(\mathrm{xz})$ & (yz) & $\begin{array}{l}\mathrm{i}=1, \\
2,7,8\end{array}$ & $\begin{array}{c}\mathrm{i}=3, \ldots, \\
6,9 \\
\ldots, 12\end{array}$ \\
\hline \multirow{5}{*}{$\begin{array}{c}A_{1} \\
+ \\
A_{2}\end{array}$} & \multirow[t]{5}{*}{$\mathrm{A}$} & \multirow[t]{5}{*}{$A_{1}$} & $2 A_{1}$ & $\otimes$ & $\otimes$ & $\otimes$ & & & & $A_{1}$ & $A_{1}$ \\
\hline & & & $2 A_{2}$ & & & & & & & $A_{2}$ & $A_{2}$ \\
\hline & & & $2 B_{1}$ & $*$ & $*$ & & & & & $B_{1}$ & $B_{1}$ \\
\hline & & & $2 B_{2}$ & & & & $*$ & & & $B_{2}$ & $B_{2}$ \\
\hline & & & $2 \mathrm{E}$ & & & & & $*$ & $*$ & & $2 \mathrm{E}$ \\
\hline \multirow[t]{5}{*}{$2 \mathrm{E}$} & \multirow[t]{5}{*}{$\mathrm{E}$} & \multirow[t]{5}{*}{$F_{2}$} & $2 A_{1}$ & * & ** & * & & & & & $2 A_{1}$ \\
\hline & & & $2 A_{2}$ & & & & & & & & $2 A_{2}$ \\
\hline & & & $2 B_{1}$ & $*$ & $*$ & & & & & & $2 B_{1}$ \\
\hline & & & $2 B_{2}$ & & & & * & & & & $2 B_{2}$ \\
\hline & & & $8 \mathrm{E}$ & & & & & $\otimes$ & $\otimes$ & $4 \mathrm{E}$ & $4 \mathrm{E}$ \\
\hline \multirow{5}{*}{$\begin{array}{c}B_{1} \\
+ \\
B_{2}\end{array}$} & \multirow[t]{5}{*}{$\mathrm{B}$} & \multirow[t]{5}{*}{ 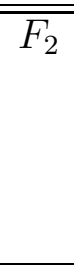 } & $2 A_{1}$ & $*$ & $*$ & $*$ & & & & $A_{1}$ & $\bar{~} \overline{A_{1}}$ \\
\hline & & & $2 A_{2}$ & & & & & & & $A_{2}$ & $A_{2}$ \\
\hline & & & $2 B_{1}$ & $*$ & $*$ & & & & & $B_{1}$ & $B_{1}$ \\
\hline & & & $2 B_{2}$ & & & & $\otimes$ & & & $B_{2}$ & $B_{2}$ \\
\hline & & & $2 \mathrm{E}$ & & & & & $*$ & $*$ & & $2 \mathrm{E}$ \\
\hline \multirow{5}{*}{$\begin{array}{c}A_{1} \\
+ \\
A_{2}\end{array}$} & \multirow[t]{5}{*}{$\mathrm{A}$} & \multirow[t]{5}{*}{$\bar{E}$} & $2 A_{1}$ & $\otimes$ & $\otimes$ & $\otimes$ & & & & $A_{1}$ & $\overline{A_{1}}$ \\
\hline & & & $2 A_{2}$ & & & & & & & $A_{2}$ & $A_{2}$ \\
\hline & & & $2 B_{1}$ & $*$ & $*$ & & & & & $B_{1}$ & $B_{1}$ \\
\hline & & & $2 B_{2}$ & & & & $*$ & & & $B_{2}$ & $B_{2}$ \\
\hline & & & $2 \mathrm{E}$ & & & & & $*$ & $*$ & & $2 \mathrm{E}$ \\
\hline \multirow{5}{*}{$\begin{array}{c}B_{1} \\
+ \\
B_{2}\end{array}$} & \multirow[t]{5}{*}{$\mathrm{B}$} & \multirow[t]{5}{*}{$E$} & $2 A_{1}$ & $*$ & $\bar{*}$ & * & & & & $A_{1}$ & $\overline{A_{1}}$ \\
\hline & & & $2 A_{2}$ & & & & & & & $A_{2}$ & $A_{2}$ \\
\hline & & & $2 B_{1}$ & $\otimes$ & $\otimes$ & & & & & $B_{1}$ & $B_{1}$ \\
\hline & & & $2 B_{2}$ & & & & $*$ & & & $B_{2}$ & $B_{2}$ \\
\hline & & & $2 \mathrm{E}$ & & & & & $*$ & $*$ & & $2 \mathrm{E}$ \\
\hline
\end{tabular}


has been discussed recently - as a transition related with ordering of $\mathrm{H}_{2} \mathrm{PO}_{4}$ groups with protons near the "upper" or the "lower" oxygen ions (with $C_{2}$ symmetry). This point of view is presented in $[10,12]$. We note that practical realization of such a model causes some difficulties due to the violations in the case of disordered state of the "ice rule" (one proton on the bond and two protons near $\mathrm{PO}_{4}$ group). On the other hand, the idea of ordering $\mathrm{H}_{2} \mathrm{PO}_{4}$ groups was not confirmed in the experiments for investigating the nuclear quadrupole resonance in $\mathrm{KH}_{2} \mathrm{PO}_{4}$ [13] as well as the isotopic frequency shift in $\mathrm{K}\left(\mathrm{D}_{x} \mathrm{H}_{1-x}\right)_{2} \mathrm{PO}_{4}$ [14]; the data obtained support the proton ordering model.

Finally, the approach proposed in this paper may be applied in the analysis of Raman scattering in other ferroelectric crystals with hydrogen bonds as well as in the crystals of another type with the phase transition of the order-disorder type. In the framework of the theory developed the interactions between electrons and lattice anharmonisms [15] may be taken into account. More detailed investigation of phonon spectrum of the crystals considered is of interest.

In particular, the systems of equations for the averaged phonon Green functions determining the shapes of the lines in Raman scattering spectra are obtained in the coherent potential approximation [4]. Configurationally splitted components of the Raman scattering lines are studied using a simple model. For $\mathrm{KH}_{2} \mathrm{PO}_{4}$ and $\mathrm{CsH}_{2} \mathrm{PO}_{4}$ type ferroelectrics, the possibility to observe experimentally the configurational splitting is discussed.

\section{References}

1. Cowley R.A. The lattice dynamics of an anharmonic crystal. // Adv. Phys., 1963, vol. 12 , No. 18, p. 421-481.

2. Stasyuk I.V., Ivankiv Ya. The specific features of Raman scattering in the orderdisorder type ferroelectrics. - In: "Sovremennye problemy statisticheskoj fiziki. Trudy Vsesoyuznoj Konferencii, L'vov, 3-5 fevralja 1987, vol. 2". Kyiv, "Naukova Dumka", 1989, p. 261-265 (in Russian).

3. Slobodyan P., Stasyuk I.V. The diagram technique for Hubbard operators. // Theor. Math. Phys., 1974, vol. 19, p. 423-429 (in Russian).

4. Ivankiv Ya., Stasyuk I.V. Configurational splitting in Raman scattering spectra in crystals with order-disorder type phase transitions. Preprint of the Institute of theoretical physics, ITP-88-80R, Kyiv, 1988, 24 p. (in Russian).

5. Slater Y.C. Theory of the transition in $\mathrm{KH}_{2} \mathrm{PO}_{4}$. // J. Chem. Phys., 1941, vol. 9, p. $16-33$.

6. Ishibashi Yo., Suzuki I. Detailed analyses and an extension of the Slater theory of the phase transitions in $\mathrm{KH}_{2} \mathrm{PO}_{4}$. // J. Phys. Soc. Jap., 1984, vol. 53, No. 1, p. 244-249.

7. Tanaka H., Tokunaga M., Tatsuzaki I. Internal modes and the local symmetry of $\mathrm{PO}_{4}$ tetrahedra in $\mathrm{K}\left(\mathrm{H}_{1-x} \mathrm{D}_{x}\right)_{2} \mathrm{PO}_{4}$ by Raman scattering. // Sol. Stat. Commun., 1984, vol.149, No. 2, p. 153-155.

8. Stasyuk I.V., Ivankiv Ya. Raman light scattering in crystals with ordered structure elements. Preprint of the Institute of theoretical physics, ITP-87-57R, Kyiv, 1987, 25 p. (in Russian). 
9. Tokunaga M., Tominaga Y., Tatsuzaki I. Order-disorder model of $\mathrm{PO}_{4}$ dipoles in RDP based on recent Raman spectroscopic studies. // Ferroelectrics, 1985, vol. 63, No. 1/4, p. $171-178$.

10. Tominaga Y., Tokunaga M., Tatsuzaki I. Scattering wave vector dependence of Raman intensity of the $\nu_{1}$ internal mode in $\mathrm{KH}_{2} \mathrm{PO}_{4}$. // Sol. Stat. Commun., 1985, vol. 54, No. 11, p. 979-980.

11. Tominaga Y., Tokunaga M., Tatsuzaki I. Dynamical mechanism of ferroelectric phase transition in $\mathrm{KH}_{2} \mathrm{PO}_{4}$ by Raman scattering study. // Jap. J. Appl. Phys., 1985, Pt. 1, vol. 24, Suppl. 24-2, p. 917-919.

12. Tokunaga M. Order-disorder model of $\mathrm{PO}_{4}$ dipoles for KDP and ADP. // Jap. J. Appl. Phys., 1985, Pt. 1, vol. 24, Suppl. 24-2, p. 908-910.

13. Blinc R. ${ }^{17} \mathrm{O} \mathrm{NQR}$ and the mechanism of the phase transition in $\mathrm{KH}_{2} \mathrm{PO}_{4}$ type $\mathrm{H}-$ bonded systems. // Z. Natur forsch., 1986, vol. 41a, p. 249-255.

14. Anachkova E., Socvatinova I. Isotopic shift and mode interaction in $\mathrm{K}\left(\mathrm{D}_{x} \mathrm{H}_{1-x}\right)_{2} \mathrm{PO}_{4}$ crystals. // Phys. status solidi (b), 1985, vol. 131, No. 2, p. K101-K105.

15. Ivankiv Ya. Polarizability operator of the dielectric crystals. // Fiz. electronika, 1985, No. 30, p. 43-47 (in Russian).

\title{
Раманівське розсіяння у кристалах з фазовими переходами типу лад-безлад
}

\author{
Я.Іванків \\ Інститут фізики конденсованих систем НАН України, \\ 79011 Львів, вул. Свєнціцького, 1 \\ Отримано 14 жовтня 1998 р.
}

На основі формалізму двочасових функцій Гріна розвинуто теорію побудови оператора поляризованості, що входить у загальний вираз для ефективного поперечного перерізу раманівського розсіяння. Представлена мікроскопічна схема використовується для дослідження сегнетоелектриків з водневими зв'язками. Ключові слова: раманівське розсіяння, фазові переходи типу
лад-безлад

PACS: $77.84 . F a, 78.30 . L y$ 
